\title{
Effective field theory as the bridge between lattice QCD and nuclear physics
}

\author{
David B. Kaplan \\ Institute for Nuclear Theory, Seattle, WA, 98195-1550, USA
}

\begin{abstract}
A confluence of theoretical and technological developments are beginning to make possible contributions to nuclear physics from lattice QCD. Effective field theory plays a critical role in these advances. I give several examples.
\end{abstract}

Keywords: nuclear physics, lattice QCD, effective field theory,chiral perturbation theory

PACS: $12.38 .-\mathrm{t}, 12.39 . \mathrm{Fe}, 12.38 . \mathrm{Gc}$

\section{INTRODUCTION}

While it is unrealistic to expect to see a solution of the structure of a uranium nucleus from QCD within our lifetimes, it is not unreasonable to predict that lattice QCD will make significant contributions to nuclear physics over the next couple of decades. Simultaneous progress in computer technology, computational algorithms, and advances in theory have made it feasible to begin such a program in lattice nuclear physics now.

The limitation one faces is the computational cost of a realistic simulation. L. Giusti presented the following formula at Lattice '06 for the cost (in Tflopsyrs) for generating gauge field configurations with dynamical Wilson fermions (http://www.physics.arizona.edu/lattice06/):

$$
\text { Cost } \sim 0.15 \cdot\left[\frac{\# \text { configs }}{1000}\right] \cdot\left[\frac{m_{q}}{20 \mathrm{MeV}}\right]^{-1} \cdot\left[\frac{V}{32 \mathrm{fm}^{4}}\right]^{\frac{5}{4}} \cdot\left[\frac{a}{0.08 \mathrm{fm}}\right]^{-6}
$$

Here $a$ is the lattice spacing, $m_{q}$ is the light quark mass, $V$ is the lattice volume.

Significant advances in algorithms have occurred in recent years, with the discovery in the 1990's of how to simulate chiral fermions [1, 2], and the improvement of methods for including light dynamical fermions (for example, the power of the mass dependence in the above formula has dropped from $m_{q}^{-6}$ to $m_{q}^{-1}$ since the development of algorithms in refs. [3, 4]). Technological advances continue unabated, and machines currently exist operating in the $10^{2}$ Tflops range, and Pflops computing will exist before long.

Nevertheless, technology plus algorithms do not by themselves add up to advances in nuclear theory in the near future because of the daunting computation costs of a realistic simulation. To avoid the disadvantages of non-chiral lattice fermions, such as the Wilson formulation, one should use domain wall or overlap fermions, incurring in the cost another factor of $\sim 100 \times$; the correct light quark masses are $m_{u} \simeq 2.5 \mathrm{MeV}$, and $m_{d} \simeq 5 \mathrm{MeV}$, not $20 \mathrm{MeV}$; the box size should be ample enough to accommodate the hadrons of interest (the Compton wavelength of the pion is about $1.4 \mathrm{fm}$, while the scattering length for the deuteron is about $5 \mathrm{fm}$ ); and the lattice spacing of the real world 
is, of course, zero. Finally, the above cost estimate only covers generation of lattice configurations; one must also account for the cost of generating quark propagators, the number of which grows factorially with the number of quarks involved - an unfortunate fact highly relevant to the study of even the smallest nuclei! Lattice QCD studies of a helium nucleus, for example, require $6 !^{2}=518,400$ quark propagator contractions. It is easy to see that a brute force approach on a Pflops machine will not provide useful information about the $\alpha$ particle at realistic quark masses.

Effective field theory is the tool that will allow us to extract useful information from available technology, giving us the ability to simulate real systems at unrealistic lattice parameters.

In particular, we will have the opportunity to learn about fundamental properties of matter which are not directly obtainable from experiment, and which are necessary inputs for reliable nuclear structure or equation-of-state calculations. These include an improved understanding of three-body forces, such as in the experimentally inaccessible $I=3 / 2$ channel, and the interactions between hyperons and nucleons. The thesis of this talk is that progress in these directions will need an intense effort by theorists in order to extract physically relevant quantities from feasible lattice calculations, and that the basic tool for this effort will be effective field theory.

Effective field theory (EFT) in all its forms is basically a perturbative expansion in the ratio of two length scales. As such, its validity requires that there be small ratios to exploit. Chiral perturbation theory has been the basic EFT exploited in continuum QCD, making use of the mass gap between the pion and the heavier hadrons. In addition, an effective field theory for nuclear physics has been in the making over the past 15 years, which incorporates an additional small ratio, the QCD length divided by the $N N$ scattering length.

What is new when working with lattice QCD is that there are a host of additional dimensionful scales which do not exist in the real world, but which can be profitably exploited. These include the lattice spacing, the lattice size, and independently varied masses for valence and sea quarks. EFT allows one to

- extrapolate to smaller quark mass than is feasible to simulate

- parametrize and correct for finite lattice spacing errors

- parametrize and correct for finite volume errors

- extract physics from "cheaper" fermions

- determine $S$-matrix elements from Euclidean simulations by measuring volume dependence of the spectrum

- extract useful physical quantities from complicated multi-hadron systems

\section{USES OF CHIRAL PERTURBATION THEORY}

\section{Quark mass extrapolation}

Chiral perturbation theory is an expansion of the Lagrangian for low energy QCD about the chiral limit, $m_{q}=0$. As such, it is obviously useful to extrapolate from lattice simulations at somewhat heavy quark masses, down to realistic quark masses. For this 
to work, the lattice quark mass has to be light enough so that the chiral expansion still converges. The chiral expansion parameter for mesonic processes is $m_{\pi}^{2} / \Lambda^{2}$, where $\Lambda \sim m_{\rho}$ is not far from $1 \mathrm{GeV}$. A light quark mass of $20 \mathrm{MeV}$, for example, corresponding to $m_{\pi} \sim 325 \mathrm{MeV}$, should be within the range of validity of chiral perturbation theory. I will not dwell on this conventional and important application of chiral perturbation theory which is widely familiar (see lectures by S. Sharpe [5] for a comprehensive introduction to lattice applications of chiral perturbation theory).

\section{Lattice spacing extrapolation}

Another application of chiral perturbation theory is to account for finite lattice spacing errors. One first matches the lattice action to the "Symanzik action" - a continuum theory with all operators allowed by the lattice symmetries, suppressed by powers of the lattice spacing $a$ appropriate to the dimension of the operator. For example, with Wilson fermions (which do not possess a chiral symmetry), the leading operators in the Symanzik action not present in continuum QCD include

- dimension-3 chiral symmetry violation: $a^{-1} \bar{q} q$

- dimension-5 chiral symmetry violation: $a \bar{q} \sigma_{\mu \nu} G_{\mu \nu} q$

- dimension-6 Lorentz violation: $a^{2} \bar{q} D_{\mu}^{3} \gamma^{\mu} q$

In order to determine the effects of finite lattice spacing on low energy hadronic physics, on can then match the Symanzik action onto a generalized chiral Lagrangian, which includes the effects of these finite lattice spacing operators [6, 7, 8]. The coefficients of these operators may be determined by making measurements at several lattice spacings, and then the extrapolation to $a=0$ may be improved.

This program is versatile and can be applied to different lattice fermion formulations. For Wilson fermion the chiral symmetry violating operators give rise to the leading $O(a)$ corrections, even when the dimension-3 operator is fine-tuned away. For staggered fermions, corrections begin at $O\left(a^{2}\right)$, but the effective theory is complicated by the presence of additional "tastes", with an approximate $S U(4)$ taste symmetry, broken by finite lattice spacing operators. The analysis of the chiral Lagrangian is simplest for chiral lattice fermions, such as domain wall or overlap fermions, which automatically avoid the $O(a)$ operators without incurring spurious fermion tastes. The computational price of dynamical chiral fermions is about a factor of 100 , which is severe.

\section{Partially quenched chiral perturbation theory}

Quark masses appear in two distinct ways in the calculation of a correlation function in lattice QCD: either in the fermion determinant, which controls the gauge field configuration one generates; or in the fermion propagators on sews together in the gauge field background to compute the desired Green function. The former is called the "sea quark mass", the latter the "valence quark mass". In the real world they are the same, but in a lattice calculation they can be different. By making the valence quark mass light 


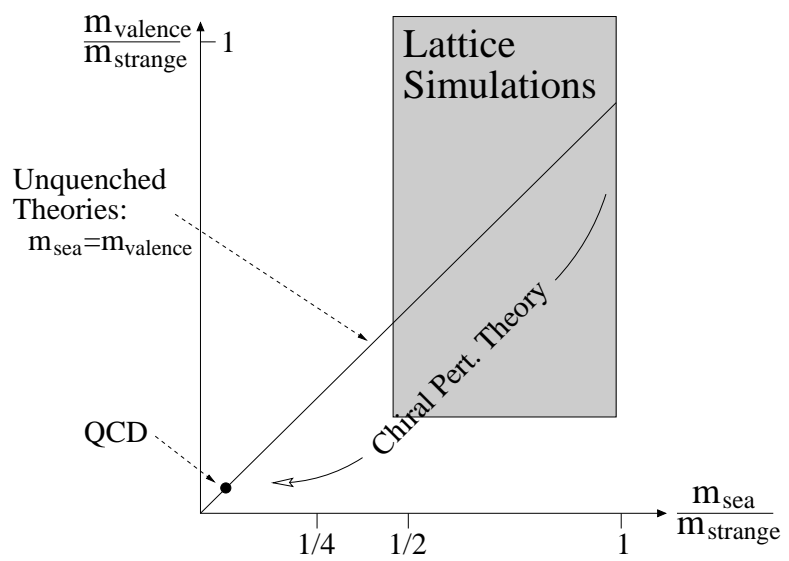

FIGURE 1. Schematic representation of parameter space in partially quenched theories, from [11].

while keeping the sea quark mass heavier, one obtains the benefits of chiral symmetry to leading order in the gauge coupling, without paying the light fermion price in generating the gauge field configurations.

One can think of this unphysical partition function arising from unphysically heavy $u$ and $d$ "sea" quarks with mass $m_{S}$, plus two flavors of light "valence" quarks with mass $m_{V}$, plus two flavors of ghosts with mass $m_{V}$ to cancel the valence quark contribution to the fermion determinant. Physical hadrons are then those made of $V V$ quarks (as well as the strange quark), in the limit $m_{V}=m_{S}$. The theory has additional unphysical mixed states of $V S$ and $S S$ content. A chiral Lagrangian can be constructed for this system, which contains new operators, but also all the operators of real QCD [9, 10]. Provided that $m_{S}$ is light enough for chiral perturbation theory to apply, by varying $m_{S}$ and $m_{V}$ independently, and by making use of generalized chiral perturbation theory for the various types of correlation functions one can compute, it is possible to isolate and measure quantities of interest in QCD, such as the Gasser-Leutwyler coefficients for $N L O$ chiral perturbation theory [11]. See fig. 1 for a picture of the expanded parameter space. For an example of how to use the partially quenched method to determine the up quark mass, see [12]; there it was shown that by computing meson masses in the combination $\left(M_{V V}^{2}+M_{S S}^{2}-2 M_{S V}^{2}\right)$ one can extract the combination of Gasser-Leutwyler coefficients $\left(2 L_{8}-L_{5}\right)$ for QCD (where $S$ and $V$ label the two propagators used).

\section{Mixed action}

There has been much work done recently with staggered fermions, employing "the fourth root trick" to reduce the number of tastes from four to one per physical quark flavor. This results in a nonlocal theory at finite lattice spacing, and there has been a controversy about whether or not the resulting lattice theory is in the same universality class as QCD and is capable of delivering an approximation to continuum QCD [13, 14]. In addition, the EFT for staggered fermions is extremely complex and difficult to work with for baryons, due to the multiplicity of tastes and taste symmetry violating operators. 
The benefits of staggered fermions are their computational cheapness, and so at least for now, they are widely employed for mesons (see, for example, [15]).

It is possible to improve the utility of staggered fermions by working with mixed actions, where the sea quarks are staggered, while the valence quarks are domain wall fermions. This approach benefits from combining the speed of staggered fermions and the chiral symmetry of domain wall fermions, and has been used in recent calculations of $g_{A}$ at the $\sim 10 \%$ level [16], the ratio $f_{\pi} / f_{K}$ to $\sim 1 \%$ [17], $\pi-\pi$ scattering [18], $K-\pi$ scattering [19], and two-nucleon properties [20]. For mixed action computations one can use the partially quenched chiral perturbation machinery described above to extract physical results from the lattice calculations [21].

\section{Volume dependence}

An additional handle on QCD provided by the lattice is the ability to manipulate the volume. Chiral perturbation theory is useful for understanding volume dependence of physical quantities, because it is the lightest modes that are most sensitive to finite volume. For a sufficiently small lattice, the zero-momentum pion modes become collective coordinates corresponding to global rotations of the chiral condensate. This occurs when $m\langle\bar{q} q\rangle V<1$, defining the $\varepsilon$-regime [22]. This regime was recently cleverly exploited to extract information about QCD in the infinite volume continuum for the $\Delta I=1 / 2$ rule [23], and for nucleon properties [24]. Both references make extensive use of chiral perturbation theory.

\section{NUCLEAR EFFECTIVE FIELD THEORY}

EFT will play a critical role in computing nuclear physics properties on the lattice. Just as for meson interactions, one would like to find strategies to measure on the lattice the coefficients of the most relevant operators which control the interactions of nucleons, and then use that effective theory to compute nuclear properties. This program requires (i) that there be a sensible EFT for the interactions of nucleons, and (ii) that one can relate lattice measurements in Euclidean space to experimentally measurable quantities, such as scattering lengths.

Nuclear effective theory was pioneered by Weinberg [25], and developed by many subsequent authors (for a review, see [26]). For low energy nucleon interactions, the pion may be considered as heavy, and the pion-less EFT developed in [27, 28, 29] consists of contact interactions $\sim C_{0}\left(N^{\dagger} N\right)^{2}+C_{2}\left(N^{\dagger} N\right)\left(N^{\dagger} \nabla^{2} N\right)+O\left(p^{4}\right) \ldots$ The momentum expansion treats $C_{2 n}=O\left(p^{n-1}\right)$ when renormalized at a scale $\mu=O(p)$, and $C_{0}$ is summed to all orders as the leading contribution, while the higher $C_{2 n}$ are inserted perturbatively. For $p \gtrsim m_{\pi} / 2$, the pion has to be included explicitly. Unfortunately, unlike the case for chiral perturbation theory, the power counting scheme for the effective theory for nucleons interacting via pions is somewhat controversial. The reason is in part because $N N$ scattering is nonperturbative, and so the actual scaling of an operator does not match its naive dimension, making it difficult to construct a consistent power counting scheme. The original Weinberg scheme suffers inconsistencies, where counterterms 
appear at higher orders than the divergences they are supposed to cancel. By working at a fixed and not too large cutoff, this problem can be swept under the rug, but this procedure in effect corresponds to constructing a model for short distance physics rather than performing a bona fide EFT calculation. This point of view is not universally accepted, and for recent contributions on various sides of the controversy see refs. [30, 31, 32, 33]. The KSW expansion [27, 28] was offered as an alternative to Weinberg's expansion, but was found not to converge well for two nucleons in the $s=1$ channel [34]. I believe that the most consistent expansion currently available is that of ref. [35], with generalizations to account for three nucleon forces [36].

There have been many notable successes of the nuclear effective theory, most remarkably at very low energy. A nice example is the isolation of the EFT coupling $L_{1 A}$, the analogue of $g_{A}$ for the axial isovector two-nucleon current. By fitting it to data one can compute the neutrino-deuteron breakup cross section, thereby reducing a major source of systematic error in the analysis of data from the Sudbury Neutrino Observatory [37].

In order to study multi-nucleon states on the lattice, one approach is to compare EFT and numerical results in Euclidean space, and determine the EFT couplings. Another approach is to compare lattice results for S-matrix elements with the predictions of the EFT. An important contribution to the problem of extracting S-matrix elements from Euclidean lattice theory was devised by Lüscher, who showed how the volume dependence of the energy for a 2-particle state in a box yields the scattering lengths [38]. A nonrelativistic formulation found in ref. [20] goes as follows: The Feynman scattering amplitude for two nucleons has the form $\mathscr{A}=(4 \pi / M) /(p \cot \delta(p)-i p)$, where $p=\sqrt{M E}, E$ being the energy in the center of mass. When formulated in a box, the energy eigenvalues correspond to zeros of $\operatorname{Re}\left[(\mathscr{A})^{-1}\right]$. So the energy eigenvalues solve

$$
0=\operatorname{Re}\left[(\mathscr{A})^{-1}\right]_{\mathrm{box}}=\operatorname{Re}\left[(\mathscr{A})^{-1}\right]_{L=\infty}+\left(\operatorname{Re}\left[(\mathscr{A})^{-1}\right]_{\mathrm{box}}-\operatorname{Re}\left[(\mathscr{A})^{-1}\right]_{L=\infty}\right) .
$$

The first term on the right is just proportional to $p \cot \delta$ in an infinite box. The second term is the difference between the bubble diagram for two nucleons scattering off each other computed in an infinite box versus computed in a finite box. This is a finite and computable function of the box size $L$ and the eigenenergy $E_{n}$, and one arrives at the formula

$$
\begin{gathered}
p_{n} \cot \delta\left(p_{n}\right)=\frac{1}{\pi L} S\left(\eta_{n}\right), \quad p_{n}=\sqrt{E_{n} M}, \quad \eta_{n}=\left(p_{n} L / 2 \pi\right)^{2}, \\
S(\eta)=\lim _{\Lambda \rightarrow \infty}\left[\sum_{|\vec{j}|<\Lambda} \frac{1}{|\vec{j}|^{2}-\eta}-4 \pi \Lambda\right],
\end{gathered}
$$

where the $\vec{j}$ are integer triplets. By measuring the energy eigenvalues for two nucleons in a box, one can then in principle solve the above equation for $\delta\left(p_{n}\right)$. A pioneering measurement of two nucleon scattering lengths using this method was performed in ref. [20], where they concluded that one would need a box of size $5-15 \mathrm{fm}$ in order to study properties of the deuteron. This is a large box, but much smaller than one would conclude directly for Lüscher's work, which would seem to indicate $L \gg a$. 
It is important to push two nucleon studies much further, technically and theoretically. Using EFT techniques, one should learn how to measure matrix elements of currents in the two-nucleon state, study hyperon-nucleon interactions, and prepare the groundwork for the study of three-nucleon states on Pflops machines.

\section{THE FRONTIER}

Lattice QCD can make substantial and useful contributions by predicting properties of baryons which are not experimentally accessible. I believe that in the foreseeable future, useful prediction are feasible in four areas:

- The masses and couplings of QCD resonances and hybrids;

- Strangeness physics, such as $\bar{K} N, Y Y$ and $Y N$ interactions, where $\bar{K}$ is the anti-kaon, $Y$ is a hyperon, and $N$ is a nucleon;

- Determination of 3-body interactions, such as in the $I=3 / 2$ channel;

- Quark mass dependence of nuclear properties.

The first category could make an important contribution to the JLab experimental program; information the second category could answer basic questions about dense matter in neutron stars, such as which hadronic channel is favored when strange quarks first appear, the $K^{-}, \Lambda$, or $\Sigma^{-}$? Accomplishing the third task would be an important milestone, whereby lattice QCD could inform the so-called $a b$ initio nuclear structure calculations and make them significantly more $a b$ initio. The fourth category could be of interest in understanding how fine-tuned is our world, and could be important in certain cosmological theories where the quark masses are dynamically determined quantities [39].

All of these projects could be very rewarding, and will require an intensive theoretical effort that further develops the 3-nucleon EFT, extends Lüscher's work to three particles in a box (where inelastic thresholds could cause problems [40]), and improves the available computational algorithms. Lattice QCD will clearly play an important role in the future progress of nuclear theory, and EFT will be a vital component of the program.

\section{ACKNOWLEDGMENTS}

I wish to thank G. Martinelli, C. Sachrajda, S. Sharpe and M. Savage for useful conversations, and to the organizers of QCHSVII for their hospitality. This work was supported by the US Department of Energy grant DE-FG02-00ER41132.

\section{REFERENCES}

1. D. B. Kaplan, Phys. Lett. B288, 342-347 (1992), hep-lat/9206013

2. H. Neuberger, Phys. Lett. B417, 141-144 (1998), hep-lat/9707022.

3. M. Luscher, JHEP 05, 052 (2003), hep-lat/ 0304007.

4. M. Luscher, Comput. Phys. Commun. 165, 199-220 (2005), hep-lat/0409106.

5. S. R. Sharpe (2006), hep-lat/0607016.

6. S. R. Sharpe, and J. Singleton, Robert L., Phys. Rev. D58, 074501 (1998), hep-lat/9804028. 
7. G. Rupak, and N. Shoresh, Phys. Rev. D66, 054503 (2002), hep-lat/0201019.

8. O. Bar, G. Rupak, and N. Shoresh, Phys. Rev. D70, 034508 (2004), hep-lat/ 0306021

9. C. W. Bernard, and M. F. L. Golterman, Phys. Rev. D49, 486-494 (1994), hep-lat/ 9306005.

10. S. R. Sharpe, and R. S. Van de Water, Phys. Rev. D69, 054027 (2004), hep-lat/ 0310012.

11. S. R. Sharpe, and N. Shoresh, Nucl. Phys. Proc. Suppl. 83, 968-970 (2000), hep-lat/9909090

12. A. G. Cohen, D. B. Kaplan, and A. E. Nelson, JHEP 11, 027 (1999), hep-lat/9909091.

13. M. Creutz (2006), hep-lat/0608020

14. S. R. Sharpe (2006), hep-lat/0610094.

15. A. S. Kronfeld, et al., PoS LAT2005, 206 (2006), hep-lat/ 0509169.

16. R. G. Edwards, et al., Phys. Rev. Lett. 96, 052001 (2006), hep-lat/ 0510062

17. S. R. Beane, P. F. Bedaque, K. Orginos, and M. J. Savage (2006), hep-lat/ 0606023.

18. S. R. Beane, P. F. Bedaque, K. Orginos, and M. J. Savage, Phys. Rev. D73, 054503 (2006), hep-lat/0506013.

19. S. R. Beane, et al. (2006), hep-lat/0607036.

20. S. R. Beane, P. F. Bedaque, K. Orginos, and M. J. Savage, Phys. Rev. Lett. 97, 012001 (2006), hep-lat/0602010

21. O. Bar, C. Bernard, G. Rupak, and N. Shoresh, Phys. Rev. D72, 054502 (2005), hep-lat/0503009.

22. J. Gasser, and H. Leutwyler, Nucl. Phys. B307, 763 (1988).

23. P. Hernandez, and M. Laine (2006), hep-lat/ 0607027.

24. W. Detmold, and M. J. Savage, Phys. Lett. B599, 32-42 (2004), hep-lat/ 0407008.

25. S. Weinberg, Phys. Lett. B251, 288-292 (1990).

26. P. F. Bedaque, and U. van Kolck, Ann. Rev. Nucl. Part. Sci. 52, 339-396 (2002), nucl-th/0203055

27. D. B. Kaplan, M. J. Savage, and M. B. Wise, Phys. Lett. B424, 390-396 (1998), nucl-th/9801034

28. D. B. Kaplan, M. J. Savage, and M. B. Wise, Nucl. Phys. B534, 329-355 (1998), nucl-th/9802075.

29. J.-W. Chen, G. Rupak, and M. J. Savage, Nucl. Phys. A653, 386-412 (1999), nucl-th/9902056.

30. A. Nogga, R. G. E. Timmermans, and U. van Kolck, Phys. Rev. (????).

31. E. Epelbaum, and U. G. Meissner (2006), nucl-th/0609037.

32. M. Rho (2006), nucl-th/0610003.

33. E. Ruiz Arriola, and M. Pavon Valderrama (2006), nucl-th/0609080

34. S. Fleming, T. Mehen, and I. W. Stewart, Nucl. Phys. A677, 313-366 (2000), nucl-th/9911001.

35. S. R. Beane, P. F. Bedaque, M. J. Savage, and U. van Kolck, Nucl. Phys. A700, 377-402 (2002), nucl-th/0104030.

36. P. F. Bedaque, G. Rupak, H. W. Griesshammer, and H.-W. Hammer, Nucl. Phys. A714, 589-610 (2003), nucl-th/0207034.

37. J.-W. Chen, K. M. Heeger, and R. G. H. Robertson, Phys. Rev. C67, 025801 (2003), nucl-th/0210073.

38. M. Luscher, Nucl. Phys. B354, 531-578 (1991).

39. S. R. Beane, and M. J. Savage, Nucl. Phys. A717, 91-103 (2003), nucl-th/0208021

40. C. J. D. Lin, G. Martinelli, C. T. Sachrajda, and M. Testa, Nucl. Phys. B619, 467-498 (2001), hep-lat/0104006. 